NASA Technical Memorandum 102099
ICOMP-89-7

\title{
Absorbing Boundary Conditions for Second-Order Hyperbolic Equations
}

(NASA-TA-102CCS) DESCEBIEG ECONLAEY
CCADITICAS FOE SECCND-CEDEE EYEEECLIC

eCOAICAS (nasa. Levis Hesearch Center)

$33 \mathbf{F}$

CSCL 12A

$\begin{array}{ll}\text { Unclas } \\ 63 / 64 & 0204409\end{array}$

บ89-22357

Hong Jiang

University of Alberta

Edmonton, Canada

and

Yau Shu Wong

Institute for Computational Mechaniç in Propulsion

Lewis Research Center

Cleveland, Ohio

April 1989

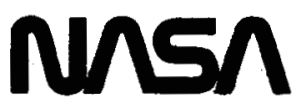




\title{
ABSORBING BOUNDARY CONDITIONS FOR SECOND-ORDER HYPERBOLIC EQUATIONS
}

\author{
HONG JIANG* \\ Department of Mathematics, University of Alberta \\ Edmonton, CANADA \\ and \\ YAU SHU WONG** \\ Institute for Computational Mechanics in Propulsion \\ NASA Lewis Research Center, Cleveland, Ohio USA \\ and \\ Department of Mathematics, University of Alberta, Edmonton, CANADA
}

\begin{abstract}
A uniform approach to construct absorbing artificial boundary conditions for secondorder linear hyperbolic equations is proposed. The nonlocal boundary condition is given by a pseudodifferential operator that annihilates travelling waves. It is obtained through the dispersion relation of the differential equation by requiring that the initial-boundary value problem admits the wave solutions travelling in one direction only. Local approximation of this global boundary condition yields an $n$ th-order differential operator. It is shown that the best approximations must be in the canonical forms which can be factorized into first-order operators. These boundary conditions are perfectly absorbing for wave packets propagating at certain group velocities.

A hierarchy of absorbing boundary conditions is derived for transonic small perturbation equations of unsteady flows. These examples illustrate that the absorbing boundary conditions are easy to derive, and the effectiveness is demonstrated by the numerical experiments.
\end{abstract}

\footnotetext{
*This work was supported by an Izaak Walton Killam Memorial Scholarship, and in part by the Natural Sciences and Engineering Research Council of Canada.

**This work was supported by the Natural Sciences and Engineering Research Council of Canada, and in part by NASA (funded under the Space Act Agreement C99066G) while the author was visiting ICOMP, NASA Lewis Research Center.
} 


\section{INTRODUCTION}

Numerical simulations of many physical processes often require calculations of solutions to partial differential equations on some infinite region. In such problems, it is essential to introduce some techniques to restrict calculations to a finite computational region. (See [1], [16] and [20].) A traditional method is the coordinates transformation, by which an infinite physical region is mapped into a finite computational domain. While this technique is effective for some steady state problems, it is inadequate for unsteady calculations arising from such applications as seismology, meteorology and transonic fluid dynamics. (See [7], [9]). An alternative is to introduce some artificial boundaries to obtain a finite region. At these artificial boundaries, some boundary conditions have to be prescribed to ensure a unique and well-posed solution. Over the years, there has been substantial interest in developing absorbing artificial boundary conditions that eliminate the nonphysical reflections, cf. [3], [4], [5], [6], [7], [8], [10], [12], [22], [24] and [25].

Using the wave equation as their model, Engquist and Majda [6, 7] proposed a pseudo-differential operator as a perfectly absorbing boundary condition. Since the dispersion relation of the wave equation admits two modes, the right-going and the left-going, which correspond to the two branches of the square root function $\pm \sqrt{1-s^{2}}$, an equation which admits only one of these modes is obtained by choosing a particular branch of the square root. This equation is then considered as the dispersion relation of an equation containing a pseudodifferential operator. Since a pseudodifferential operator is nonlocal in both time and space, this boundary condition is not numerically useful in practice. Engquist and Majda then used Padé-approximation of the square root $\sqrt{1-s^{2}}$ to arrive at a hierarchy of local boundary conditions given by differential operators. These boundary conditions are perfectly absorbing at normal incidence.

Higdon [13] proposed a process by which Engquist-Majda boundary conditions can be generalized to be perfectly absorbing at some arbitrary angles of incidence. In this process, he was able to show that for certain approximations, a differential operator in 
Engquist-Majda boundary conditions can be put into a canonical form. It can be factorized into differential operators of first-order, with each factor annihilating waves at a specific angle of incidence. This canonical form has theoretical and practical advantages: it does a great deal to simplify stability analysis and numerical implementation [13]. Also this characterization of absorbing boundary condition highlights the relation between EngquistMajda boundary conditions and Bayliss-Turkel boundary conditions [5].

The purpose of this paper is threefold:

1) We propose another approach, which is related to that of Engquist and Majda in [6] and [7], to construct local absorbing boundary conditions. In their process of rational approximation of the square root function, Engquist and Majda have used different strategies for different equations, namely, the wave equation in [6] and [7] and the transonic small disturbance equation in [8]. In our approach, a fairly uniform strategy of rational approximation is applied, and the absorbing boundary conditions can be automatically generated as long as the dispersion relation is known.

2) As was pointed out in [13], the factorization theorem of Higdon applies only to certain rational approximations for wave equations. We will generalize this factorization theorem to show that a larger class of absorbing boundary conditions can be in fact put into Higdon's canonical form. The use of this form will have the advantages both in analysis and implementation, especially when higher-order conditions are concerned. When applied to such equations as the wave equation and the dispersive Klein-Gordon equation, our method yields the same results as established in [13].

3) High-order absorbing boundary conditions often involve some optimization parameters. Our approach of constructing absorbing boundary conditions provides a natural link between these parameters and the group velocities of wave solutions. We will show that the optimal absorbing boundary conditions are those which are perfectly absorbing at ccrtain group velocities, with each factor annihilating the wave packets propagating at a specific group velocity. This physical interpretation is helpful in determining the 
optimization parameters.

In section 2, we will derive perfectly absorbing boundary conditions for the travelling wave solutions. Its local approximation will be considered in section 3 . Also in section 3 , we will describe the general characterization of the absorbing boundary conditions. As applications, we will derive a hierarchy of absorbing boundary conditions for the transonic small disturbance equations in section 4 . Finally, in section 5 , we will compare by numerical experiments our boundary conditions with Engquist-Majda boundary conditions of [8].

\section{PERFECTLY ABSORBING BOUNDARY CONDITION}

In this section, we derive a global boundary condition which annihilates travelling waves of a general second-order hyperbolic equation. In fact, our global absorbing boundary condition applies to any linear hyperbolic equations with constant coefficients for which a dispersion relation is known.

We consider the initial-boundary value problem (IBVP):

$$
\begin{gathered}
P\left(\frac{\partial}{\partial t} ; D\right) u=0, \quad t>0, \quad x_{1} \geq 0 \\
u\left(t ; 0, x_{-}\right)=g\left(t ; x_{-}\right), \quad t>0, \quad x_{-} \in \mathbb{R}^{n-1}, \\
u \equiv 0, \quad t \leq 0,
\end{gathered}
$$

where $D=\left(\frac{\partial}{\partial x_{1}}, \ldots, \frac{\partial}{\partial x_{n}}\right)$ and $x_{-}=\left(x_{2}, \ldots, x_{n}\right) . \quad P$ is a homogeneous polynomial of degree two of $n+1$ variables with constant coefficients. Furthermore, we assume $P$ is hyperbolic in the direction of $t$ (see [19]) and that $g$ has a compact support. Problem (2.1) must be solved over a region which is bounded in $x_{1}$, e.g., $\Omega=\left\{\left(x_{1}, \ldots, x_{n}\right) \mid x_{1} \leq a\right\}$.

By an absorbing boundary condition $B u=0$ at $x_{1}=a$, it is meant that the solution 
of (2.1) can be well approximated by the solution of the following problem.

$$
\begin{aligned}
& P\left(\frac{\partial}{\partial t} ; D\right) u=0, t>0, \quad 0 \leq x_{1} \leq a \\
& u\left(t ; 0, x_{-}\right)=g\left(t ; x_{-}\right), t>0, \quad x_{-} \in \mathbb{R}^{n-1}, \\
& B u=0, x_{1}=a, \quad t>0, \quad x_{-} \in \mathbb{R}^{n-1} \\
& u \equiv 0, \quad t \leq 0 .
\end{aligned}
$$

Let $(\tau, \xi) \in \mathbb{R}^{n+1}$ be the dual of $(t, x)$ and $\xi_{-} \in \mathbb{R}^{n-1}$ be the dual of $x_{-}$. Equation (2.1) admits plane wave solutions of the form

$$
u(t ; x)=e^{i(\tau t+\xi \cdot x)}
$$

for $(\tau, \xi) \in \mathbb{R}^{n+1}$, provided the dispersion relation

$$
\sigma(\tau ; \xi) \equiv P(i \tau ; i \xi)=0
$$

is satisfied, where $i \xi=\left(i \xi_{1}, \ldots, i \xi_{n}\right)$. The group velocity of (2.3) with which the energy of the wave packet is propagated is equal to (see [26])

$$
\vec{C}=-\nabla_{\xi} \cdot \tau
$$

For a given frequency $\tau \in \mathbb{R}$ and a wavenumber $\xi_{-} \in \mathbb{R}^{n-1}$, we assume that the equation in (2.1) admits the plane wave solutions (2.3) which propagate in both the positive and the negative directions of the $x_{1}$-axis. Thus for some $\left(\tau, \xi_{-}\right) \in \mathbb{R}^{n},(2.4)$ has two roots $\xi_{1}^{+}, \xi_{1}^{-} \in \mathbb{R}$ such that the $x_{1}$-components of group velocities $V_{x_{1}}$ are positive and negative respectively, i.e.,

$$
V_{x_{1}}\left(\xi_{1}^{-}\right) \equiv-\frac{\partial \tau}{\partial \xi_{1}}\left(\tau ; \xi_{1}^{-}, \xi_{-}\right) \leq 0 \leq-\frac{\partial \tau}{\partial \xi_{1}}\left(\tau ; \xi_{1}^{+}, \xi_{-}\right) \equiv V_{x_{1}}\left(\xi_{1}^{+}\right)
$$

The travelling waves which propagate in the positive $x_{1}$-direction has the form

$$
u(t ; x)=e^{i\left(\tau t+\xi_{1}^{+} x_{1}+\xi_{-} \cdot x_{-}\right)} .
$$


It follows that a boundary condition that annihilates the right-going wave solutions (2.5) can be written as

$$
-\frac{\partial \tau}{\partial \xi_{1}}(\tau ; \xi)=\left|-\frac{\partial \tau}{\partial \xi_{1}}(\tau ; \xi)\right| .
$$

From (2.4), the implicit function theorem leads to

$$
-\frac{\partial \tau}{\partial \xi_{1}}=\frac{\sigma_{\xi_{1}}}{\sigma_{\tau}}
$$

So the perfectly absorbing boundary condition at $x_{1}=a$ in the absence of evanescent waves is given by

$$
\frac{\sigma_{\xi_{1}}}{\sigma_{\tau}}(\tau ; \xi)=\left|\frac{\sigma_{\xi_{1}}}{\sigma_{\tau}}(\tau ; \xi)\right|
$$

or

$$
\frac{\sigma_{\xi_{1}}}{\sigma_{\tau}}(\tau ; \xi)-\left|\frac{\sigma_{\xi_{1}}}{\sigma_{\tau}}(\tau ; \xi)\right|=0
$$

The left hand side of (2.6) can be regarded as the symbol of a pseudo-differential operator which can be expressed in terms of a Fourier integral. This operator requires the information of the solution away from the boundary $x_{1}=a$. An alternate definition of the boundary condition corresponding to (2.6) can be formulated as follows.

For $u(t ; x)=e^{i(r t+\xi \cdot x)}$, define $B^{*}$ by

$$
B^{*} u=\left\{\frac{\sigma_{\xi_{1}}}{\sigma_{\tau}}(\tau ; \xi)-\left|\frac{\sigma_{\xi_{1}}}{\sigma_{\tau}}(\tau ; \xi)\right|\right\} e^{i(\tau t+\xi \cdot x)}
$$

For more general solution, the Fourier transform $\hat{u}\left(\tau ; x_{1}, \xi_{-}\right)$of $u$ with respect to $t$ and $x_{-}$can be decomposed into a sum of two terms

$$
\hat{u}\left(\tau ; x_{1}, \xi_{-}\right)=f^{+}\left(\tau ; \xi_{-}\right) e^{i \xi_{1}^{+} x_{1}}+f^{-}\left(\tau ; \xi_{-}\right) e^{i \xi_{1}^{-} x_{1}}
$$

Then the action of $B^{*}$ on $u$ is

$$
\begin{aligned}
B^{*} u & =\iint\left\{\frac{\sigma_{\xi_{1}}}{\sigma_{\tau}}\left(\tau ; \xi_{1}^{+}, \xi_{-}\right)-\left|\frac{\sigma_{\xi_{1}}}{\sigma_{\tau}}\left(\tau ; \xi_{1}^{+}, \xi_{-}\right)\right|\right\} f^{+}\left(\tau ; \xi_{-}\right) e^{i\left(\tau t+\xi_{1}^{+} x_{1}+\xi_{-} \cdot x_{-}\right)} d \tau d \xi_{-} \\
& +\iint\left\{\frac{\sigma_{\xi_{1}}}{\sigma_{\tau}}\left(\tau ; \xi_{1}^{-}, \xi_{-}\right)-\left|\frac{\sigma_{\xi_{1}}}{\sigma_{\tau}}\left(\tau ; \xi_{1}^{-}, \xi_{-}\right)\right|\right\} f^{-}\left(\tau ; \xi_{-}\right) e^{i\left(\tau t+\xi_{1}^{-} x_{1}+\xi_{-} \cdot x_{-}\right)} d \tau d \xi_{-}
\end{aligned}
$$


We can show that if the $I B V P(2.1)$ generates travelling waves only, i.e., the Fourier transform $\hat{g}$ of $g$ satisfies

$$
\operatorname{supp} \hat{g} \subseteq \mathcal{S} \equiv\left\{\left(\tau, \xi_{-}\right) \in \mathbb{R}^{n} \mid \quad(2.4) \text { has only real roots } \xi_{1}^{+}, \xi_{1}^{-}\right\}
$$

then $B^{*}$ defined in (2.8) annihilates the exact solution of (2.1).

By using the Fourier-Laplace transform, the solution of (2.1) is given by

$$
u(t ; x)=\iint e^{i\left(\tau t+\xi_{1}^{+} x_{1}+\xi_{-} \cdot x_{-}\right)} \hat{g}\left(\tau ; \xi_{-}\right) d \tau d \xi_{-} .
$$

In the above integral, $\xi_{1}^{+}=\xi^{+}\left(\tau ; \xi_{-}\right)$is the root of (2.4) which satisfies $\operatorname{Im} \xi_{1}^{+}>0$ for each $\left(\tau ; \xi_{-}\right) \in \mathbb{C} \times \mathbb{R}^{n-1}$ with $\operatorname{Im} \tau<0$. For $\tau \in \mathbb{R}, \xi_{1}^{+}\left(\tau ; \xi_{-}\right)$is taken as the limit in the lower-half plane $\operatorname{Im} \tau<0$. It was shown in [14] that if $\xi_{1}^{+} \in \mathbb{R}$, then $V_{x_{1}}\left(\xi_{1}^{+}\right) \geq 0$, i.e., the positive group velocity in the $x_{1}$-direction.

Decompose this solution into (2.7), the result is $f^{+}=\hat{g}$ and $f^{-}=0$. Then substitute it into (2.8). The first integral in (2.8) vanishes by the relation $\frac{\sigma_{\xi_{2}}}{\sigma_{\tau}}\left(\tau ; \xi_{1}^{+}, \xi_{-}\right)=$ $\left|\frac{\sigma_{\xi_{1}}}{\sigma_{\tau}}\left(\tau ; \xi_{1}^{+}, \xi_{-}\right)\right|$and so does the second integral because $f^{-}=0$. This leads to $B^{*} u=0$. Thus the solution of (2.1) is a solution of (2.2) if $B=B^{*}$.

The solution of (2.2) is also unique when $B=B^{*}$. The boundary condition $B^{*} u=0$ implies $f^{-}\left(\tau ; \xi_{-}\right)=0$, because $\frac{\sigma_{\xi_{1}}}{\sigma_{r}}\left(\tau ; \xi_{1}^{-}, \xi_{-}\right) \neq\left|\frac{\sigma_{\xi_{1}}}{\sigma_{\tau}}\left(\tau ; \xi_{1}^{-}, \xi_{-}\right)\right|$for each $\left(\tau ; \xi_{-}\right)$. This shows that $u$ is a solution of (2.1) if and only if it is a solution of (2.2).

Therefore the boundary condition $B^{*}=0$, or equivalently (2.6), is a perfectly absorbing boundary condition. This boundary condition, however, is nonlocal in both time and space due to the presence of the absolute value function in (2.6), hence not useful in practice. In order to implement this condition numerically, it must be replaced by some local boundary conditions resulting from the rational approximations of the absolute value function. Such approximations will be discussed in section 3, but here it is probably worthwhile to mention a connection between (2.6) and the perfectly absorbing boundary condition derived by Engquist and Majda in [6]. The global boundary condition of [6] 
involves the square root function which also has to be approximated by some rational functions. According to Newman [18], the best rational approximations of the absolute value function $|x|$ and the square root $\sqrt{x}$ have the same order of accuracy. In this sense, if (2.6) is approximated by a good rational function, the localized boundary conditions resulting from (2.6) are as accurate as those of [6] and [7].

\section{CHARACTERIZATION OF HIGH-ORDER ABSORBING BOUNDARY CONDITIONS}

We now consider the local approximations of the perfectly absorbing boundary condition (2.6). In designing an absorbing boundary condition two properties must be considered: it should minimize the amplitudes of the waves reflected from the artificial boundary so that the solution of (2.2) closely approximates the free-space solution of (2.1), and it must also be a well-posed condition to guarantee a unique and well-posed solution to the differential equation. To study the absorption property of a boundary condition $B u=0$ with its dispersion relation $B(\tau, \xi)=0$, we consider the wave solutions of the form

$$
u(t ; x)=e^{i\left(\tau t+\xi_{1}^{+} x_{1}+\xi_{-} \cdot x_{-}\right)}+r e^{i\left(\tau t+\xi_{1}^{-} x_{1}+\xi_{-} \cdot x_{-}\right)} .
$$

Substitution of $u$ into the boundary condition $B u=0$ at $x_{1}=a$ yields

$$
B\left(\tau ; \xi_{1}^{+}, \xi_{-}\right)+r e^{i\left(\xi_{1}^{-}-\xi_{1}^{+}\right) a} B\left(\tau ; \xi_{1}^{-}, \xi_{-}\right)=0
$$

Solving for $r$ from the above equation, the result is

$$
r=-\frac{B\left(\tau ; \xi_{1}^{+}, \xi_{-}\right)}{B\left(\tau ; \xi_{1}^{-}, \xi_{-}\right)} e^{i\left(\xi_{1}^{+}-\xi_{1}^{-}\right) a}
$$

Then the reflection coefficient of the boundary condition $B u=0$ is defined by

$$
R_{B}\left(\tau ; \xi_{-}\right)=|r|=\left|\frac{B\left(\tau ; \xi_{1}^{+}, \xi_{-}\right)}{B\left(\tau ; \xi_{1}^{-}, \xi_{-}\right)}\right|
$$


The reflection coefficient is the amplitude of the reflected wave. Therefore a perfectly absorbing boundary condition must yield a reflection coefficient equal to 0 for all frequencies, e.g., the boundary condition (2.0̂). This is, in general, impossible to achieve for a local boundary condition. Hence, we hope to build a local boundary condition for which $R_{B}$ is as small as possible.

A theory to determine the well-posedness of an $I B V P$ has been developed by Kreiss [17] and by Sakamoto [21]. The following criterion of well-posedness can be found in [7] and [24].

\section{WELL-POSEDNESS CRITERION.}

The IBVP (2.2) is well-posed if and only if

$$
\begin{aligned}
& \sigma\left(\tau ; \xi_{1}, \xi_{-}\right)=0, \\
& B\left(\tau ; \xi_{1}, \xi_{-}\right)=0,
\end{aligned}
$$

has no eigenvalues and generalized eigenvalues.

An eigenvalue of (3.1) is defined as $\left(\tau ; \xi_{1}, \xi_{-}\right) \in \mathbb{C} \times \mathbb{C} \times \mathbb{R}^{n-1}$, which satisfies (3.1) and $\operatorname{Im} \tau \leq 0$ and $\operatorname{Im} \xi_{1}<0$.

A generalized eigenvalue of $(3.1)$ is defined as $\left(\tau ; \xi_{1}, \xi_{-}\right) \in \mathbb{F}^{n+1}$ with $\left(\tau ; \xi_{1}, \xi_{-}\right) \neq$ $(0,0,0)$, which satisfies (3.1) and

$$
V_{x_{1}}\left(\xi_{1}\right)=\frac{\sigma_{\xi_{1}}\left(\tau ; \xi_{1}, \xi_{-}\right)}{\sigma_{\tau}\left(\tau ; \xi_{1}, \xi_{-}\right)} \leq 0
$$

Discussions and interpretations of this criterion can be found in [14] and [24], and in [23] for the analogue of difference equations.

In order to obtain a local boundary condition approximating (2.6), the absolute value function in (2.6) must be approximated by a rational function. By making a rational transform if necessary, we may assume that $V_{x_{1}} \in[-1,1]$.

Let $r(x)=\frac{p_{m}(x)}{q_{n}(x)}$ of order $(m, n)$ be a rational approximation to $|x|$ on interval $[-1,1]$, then (2.6) can be approximated by

$$
\frac{\sigma_{\xi_{1}}}{\sigma_{\tau}}=r\left(\frac{\sigma_{\xi_{1}}}{\sigma_{\tau}}\right)=\frac{p_{m}\left(\sigma_{\xi_{1}} / \sigma_{\tau}\right)}{q_{n}\left(\sigma_{\xi_{1}} / \sigma_{\tau}\right)},
$$


or

$$
\sigma_{\xi_{1}} \sigma_{\tau}^{d-1} q_{n}\left(\frac{\sigma_{\xi_{1}}}{\sigma_{\tau}}\right)-\sigma_{\tau}^{d} p_{m}\left(\frac{\sigma_{\xi_{1}}}{\sigma_{\tau}}\right)=0, \quad d=\max \{m, n+1\}
$$

The left hand side of (3.3) is a homogeneous polynomial of degree $d$ in $(\tau, \xi)$, hence (3.3) is the dispersion relation of a differential equation.

An obvious choice of $r(x)$ seems to be the one, $r^{*}(x)$, obtained by Newman in [18], which has an accuracy of order $e^{-c \sqrt{d}}$. This approximation, however, results in a boundary condition that is ill-posed, because $r^{*}(0)=0$; hence, (3.3) admits a generalized eigenvalue. Although adding a small positive constant to $r^{*}$ would rule out all generalized eigenvalues while still maintaining roughly the same accuracy, it is not clear whether the resulting boundary condition admits other eigenvalues. Rather than looking for a function approximating $|x|$, we will instead take another approach due to Higdon [13], in which we study the necessary form of a well-posed boundary condition with the smallest reflection coefficient.

Equation (3.2) can be expressed as

$$
Q\left(\frac{\sigma_{\xi_{1}}}{\sigma_{\tau}}\right)=0
$$

where $Q$ is a polynomial of degree $d=\max \{m, n+1\}$ with real coefficients, which can be factorized as

$$
Q\left(\frac{\sigma_{\xi_{1}}}{\sigma_{\tau}}\right)=\prod_{j=1}^{d_{1}}\left(\frac{\sigma_{\xi_{1}}}{\sigma_{\tau}}-v_{j}\right) \prod_{j=d_{1}+1}^{\frac{d+d_{1}}{2}}\left[\left(\frac{\sigma_{\xi_{1}}}{\sigma_{\tau}}-w_{j}\right)^{2}+\hat{w}_{j}^{2}\right]=0
$$

with $v_{j}, w_{j}, \hat{w}_{j} \in \mathbb{R}$. For any polynomial (3.4), there exists a corresponding boundary condition whose dispersion relation is given by

$$
\sigma_{\tau}^{d} Q\left(\frac{\sigma_{\xi_{1}}}{\sigma_{\tau}}\right)=0
$$

The boundary condition corresponding to (3.5) will also be referred as boundary condition $Q$ of order $d$. 
The reflection coefficient of (3.5) is given by

$$
\begin{aligned}
R_{Q}=\prod_{j=1}^{d_{1}} \frac{\sigma_{\xi_{1}}\left(\xi_{1}^{+}\right)-v_{j} \sigma_{\tau}\left(\xi_{1}^{+}\right)}{\sigma_{\xi_{1}}\left(\xi_{1}^{-}\right)-v_{j} \sigma_{\tau}\left(\xi_{1}^{-}\right)} \\
\quad \times \prod_{j=d_{1}+1}^{\frac{d+d_{1}}{2}} \frac{\left(\sigma_{\xi_{1}}\left(\xi_{1}^{+}\right)-w_{j} \sigma_{\tau}\left(\xi_{1}^{+}\right)\right)^{2}+\hat{w}_{j}^{2} \sigma_{\tau}^{2}\left(\xi_{1}^{+}\right)}{\left(\sigma_{\xi_{1}}\left(\xi_{1}^{-}\right)-w_{j} \sigma_{\tau}\left(\xi_{1}^{-}\right)\right)^{2}+\hat{w}_{j}^{2} \sigma_{\tau}^{2}\left(\xi_{1}^{-}\right)}
\end{aligned}
$$

with obvious independent variables omitted.

Now, we assume $V_{x_{1}}=\frac{\sigma_{\xi_{1}}}{\sigma_{r}}(\tau, \xi) \in[\alpha, \beta]$ for all $\left(\tau, \xi_{-}\right) \in \mathcal{S}$ and $\xi \in \mathbb{R}$ such that $\sigma(\tau, \xi)=0$, and $-\infty<\alpha<0<\beta<+\infty$; that is, the $x_{1}$-component of group velocity has the lower bound $\alpha$ and the upper bound $\beta$.

Definition 3.1. Given $\varepsilon: 0<\varepsilon<\beta$, let

$$
\begin{aligned}
& \mathbb{Q}_{d}=\{Q: \text { real polynomials of degree } d\} \\
& \mathcal{S}_{\varepsilon}=\left\{\left(\tau, \xi_{-}\right) \in \mathbb{R}^{n} \mid \varepsilon \leq \frac{\sigma_{\xi_{1}}\left(\tau ; \xi_{1}^{+}, \xi_{-}\right)}{\sigma_{\tau}\left(\tau ; \xi_{1}^{+}, \xi_{-}\right)} \leq \beta\right\} .
\end{aligned}
$$

A boundary condition $Q^{*} \in \mathbb{Q}_{d}$ is said to be $\mathcal{L}_{\varepsilon}^{\infty}$-best boundary condition of order $d$ if

$$
\max _{\left(\tau, \xi_{-}\right) \in \mathcal{S}_{\boldsymbol{c}}} R_{Q^{*}}\left(\tau, \xi_{-}\right)=\min _{Q \in \mathbb{Q}_{d}}\left\{\max _{\left(\tau, \xi_{-}\right) \in \mathcal{S}_{\boldsymbol{c}}} R_{Q}\left(\tau, \xi_{-}\right)\right\},
$$

where $R_{Q}$ is the reflection coefficient of the boundary condition $Q$.

$\mathcal{L}_{\varepsilon}^{\infty}$-best boundary conditions are the optimal boundary conditions of a fixed degree, in the sense that they minimize the reflection coefficient over all travelling waves of propagating speed in the range $[\varepsilon, \beta]$. In applications, during the time interval of interest, the waves with the slow speed $\left(V_{x_{1}} \in[0, \varepsilon)\right)$ will not travel far enough to reach the boundary.

The following definition, due to Wagatha [25], takes into consideration the wave modes of every speed in $(0, \beta]$.

Definition 3.2. Let $\rho \in \mathcal{L}_{[0, \beta]}^{1}$ with $\rho \geq 0$ and

$$
\int_{0}^{\beta} \rho(v) d v=1
$$


An absorbing boundary condition $Q^{*}$ is said to be $\mathcal{L}^{1}$-best boundary condition of order $d$ if

$$
\int_{0}^{\beta} \rho\left(V_{x_{1}}\right) R_{Q^{*}}\left(V_{x_{1}}\right) d V_{x_{1}}=\min _{Q \in \mathbb{Q}_{d}} \int_{0}^{\beta} \rho\left(V_{x_{1}}\right) R_{Q}\left(V_{x_{1}}\right) d V_{x_{1}} .
$$

The $\mathcal{L}^{2}$-best boundary condition is similarly defined by replacing $R_{Q}$ by $R_{Q}^{2}$ in the above integral.

The following theorems characterize the best boundary conditions.

TheOREM 3.3. Let $\varepsilon>0$. If $Q^{*}$ is a well-posed $\mathcal{L}_{\varepsilon}^{\infty}$-best boundary condition, then

$$
Q^{*}(x)=\prod_{j=1}^{d}\left(x-v_{j}\right)
$$

Furthermore, $v_{j} \in[\varepsilon, \beta]$.

THEOREM 3.4. Let $Q^{*}$ be a well-posed $\mathcal{L}^{1}$ (or $\mathcal{L}^{2}$ )-best boundary condition. Then (3.6) holds and $v_{j} \in(0, \beta]$.

The proofs of the theorems 3.3 and 3.4 are identical; we prove Theorem 3.3 only.

Proof of TheOREM 3.3:

Since $Q^{*}$ is a polynomial of degree $d$, it can be factorized as

$$
Q^{*}(x)=\prod_{j=1}^{d_{1}}\left(x-v_{j}\right) \prod_{j=d_{1}+1}^{\frac{d+d_{1}}{2}}\left[\left(x-w_{j}\right)^{2}+\hat{w}_{j}^{2}\right] .
$$

(a) We first show that if $Q^{*}$ gives rise to an $\mathcal{L}_{\varepsilon}^{\infty}$-best boundary condition, then the quadratic factors must disappear in (3.7), i.e., $d_{1}=d$. This part of the proof was provided by one of the referees.

We consider a quadratic factor $\left(x-w_{j}\right)^{2}+\hat{w}_{j}^{2}$ and its corresponding reflection coefficient

$$
\begin{aligned}
R_{j} & =\left|\frac{\left(\sigma_{\xi_{1}}\left(\xi_{1}^{+}\right)-w_{j} \sigma_{\tau}\left(\xi_{1}^{+}\right)\right)^{2}+\hat{w}_{j}^{2} \sigma_{\tau}^{2}\left(\xi_{1}^{+}\right)}{\left(\sigma_{\xi_{1}}\left(\xi_{1}^{-}\right)-w_{j} \sigma_{\tau}\left(\xi_{1}^{-}\right)\right)^{2}+\hat{w}_{j}^{2} \sigma_{\tau}^{2}\left(\xi_{1}^{-}\right)}\right| \\
& =\hat{R}_{j} c^{2}
\end{aligned}
$$


where

$$
\hat{R}_{j}=\frac{\left(x^{+}-w_{j}\right)^{2}+\hat{w}_{j}^{2}}{\left(x^{-}-w_{j}\right)^{2}+\hat{w}_{j}^{2}}
$$

$c=\frac{\sigma_{\tau}\left(\xi_{1}^{+}\right)}{\sigma_{r}\left(\xi_{1}^{-}\right)}$and $x^{ \pm}=\frac{\sigma_{\xi_{1}}}{\sigma_{r}}\left(\xi_{1}^{ \pm}\right)$. By assumption,

$$
\alpha \leq x^{-} \leq 0<\varepsilon \leq x^{+} \leq \beta .
$$

It is easy to verify, by expanding the both sides, that

$$
\left(x^{+}-\sqrt{w_{j}^{2}+\hat{w}_{j}^{2}}\right)^{2} \leq\left(x^{+}-w_{j}\right)^{2}+\hat{w}_{j}^{2}
$$

and

$$
\left(x^{-}-\sqrt{w_{j}^{2}+\hat{w}_{j}^{2}}\right)^{2} \geq\left(x^{-}-w_{j}\right)^{2}+\hat{w}_{j}^{?} .
$$

This implies

$$
\hat{R}_{j} \geq \frac{\left(x^{+}-\sqrt{w_{j}^{2}+\hat{w}_{j}^{2}}\right)^{2}}{\left(x^{-}-\sqrt{w_{j}^{2}+\hat{w}_{j}^{2}}\right)^{2}} \text { for all } x^{-}, x^{+} .
$$

The equality in the above holds only if $\hat{w}_{j}=0$. But because $Q^{*}$ is optimal, the value of $\hat{R}_{j}$ can not be reduced, hence one concludes $\hat{w}_{j}=0$. In that case, this quadratic factor is in fact a product of two linear factors. This shows that (3.6) holds.

(b) We now consider a linear factor $\left(x-v_{j}\right)$ of (3.6) and show that if $v_{j} \notin[\varepsilon, \beta]$, then either the condition (3.6) fails the well-posedness criterion, or it is not optimal.

If $v_{j} \in[\alpha, 0]$, then the condition (3.6) is not well-posed because there exists a generalized eigenvalue. Now we consider the reflection coefficient corresponding to this factor.

$$
R_{j}=\left|\frac{x^{+}-v_{j}}{x^{-}-v_{j}}\right| c
$$

where $x^{ \pm}$and $c$ are defined in part (a).

It is easy to verify that if $v_{j} \in(-\infty, \alpha) \cup(\beta,+\infty)$, then

$$
\left|\frac{x^{+}-v_{j}}{x^{-}-v_{j}}\right|>\left|\frac{x^{+}-\beta}{x^{-}-\beta}\right|, \quad \text { for all } x^{-}, x^{+} \text {. }
$$

Also, if $v_{j} \in(0, \varepsilon)$, then

$$
\left|\frac{x^{+}-v_{j}}{x^{-}-v_{j}}\right|>\left|\frac{x^{+}-\varepsilon}{x^{-}-\varepsilon}\right|, \quad \text { for all } x^{-}, x^{+} .
$$

Therefore $v_{j} \in[\varepsilon, \beta]$ for $Q^{*}$ to be optimal. This complete the proof. 
TIIEOREm 3.5. Let $Q_{d}^{*}$ be a well-posed $\mathcal{L}_{\varepsilon}^{\infty}$-best boundary condition. If

$$
\left|\sigma_{\tau}\left(\tau ; \xi_{1}^{+}, \xi_{-}\right)\right| \leq\left|\sigma_{\tau}\left(\tau ; \xi_{1}^{-}, \xi_{-}\right)\right|
$$

for all $\left(\tau ; \xi_{-}\right) \in \mathcal{S}_{\varepsilon}$, then $R_{Q_{d}^{*}}<1$ for all $\left(\tau ; \xi_{-}\right) \in \mathcal{S}_{\varepsilon}$ and

$$
\lim _{d \rightarrow+\infty} R_{Q_{d}^{*}}=0, \quad \text { for all }\left(\tau ; \xi_{-}\right) \in \mathcal{S}_{\varepsilon}
$$

Proof: Let

$$
Q_{d}(x)=\prod_{j=1}^{d}(x-2 \beta),
$$

then $R_{Q_{d}}<1$ and $\lim _{d \rightarrow+\infty} R_{Q_{d}}=0$. The theorem follows from $R_{Q_{d}^{*}} \leq R_{Q_{d}}$.

From Theorem 3.3, the $d$ th order $\mathcal{L}_{\varepsilon}^{\infty}\left(\mathcal{L}^{1}\right.$ or $\left.\mathcal{L}^{2}\right)$-best condition for the problem (2.1) whose dispersion relation is $\sigma(\tau ; \xi)=0$ must have the form

$$
\prod_{j=1}^{d}\left\{\sigma_{\xi_{1}}\left(\frac{\partial}{\partial t} ; D\right)-v_{j} \sigma_{\tau}\left(\frac{\partial}{\partial t} ; D\right)\right\} u=0
$$

where $D=\left(\frac{\partial}{\partial x_{1}}, \ldots, \frac{\partial}{\partial x_{n}}\right), v_{j} \in[\varepsilon, \beta]($ or $(0, \beta))$. In particular, the $\mathcal{L}^{1}$ (or $\mathcal{L}^{2}$ )-best boundary conditions can be generated automatically with the help of a symbolic manipulation program in the following manner. First, the derivatives $\sigma_{\xi_{1}}, \sigma_{\tau}$ are calculated from the dispersion relation $\sigma(\tau ; \xi)=0$. Then the parameters $v_{j}$ can be approximated through a numerical process to minimize the integral in Definition 3.2. Finally, analysis for wellposedness can be carried out for each factor of (3.8), which is simple as shown in the next section.

\section{ABSORBING BOUNDARY CONDITIONS FOR A CLASS OF TRAN- SONIC SMALL DISTURBANCE EQUATIONS OF UNSTEADY FLOWS}

In their paper [8], Engquist and Majda derived absorbing boundary conditions up to the second-order for the unsteady transonic small disturbance equation of low reduced 
frequencies. Their derivation, based on the framework of their earlier work [7], did not, however, provide a uniform approach in constructing the local boundary conditions. Different strategies had to be used to approximate the square root while handling the sidewall conditions. Furthermore, if a higher-order approximation is required, their method leads to two unpleasant difficulties: the determination of well-posedness and the selection of the parameters. Although general guidelines for selecting the parameters were given, these guidelines do not have a direct physical interpretation.

In this section, we will recreate the artificial boundary conditions for the transonic small disturbance equation of low reduced frequencies treated in [8]. From our approach, a connection between absorption properties and group velocities of the interior disturbance is revealed. Boundary conditions for the transonic small disturbance equation of full frequencies will also be considered.

\subsection{Equation of Low Reduced Frequencies}

By a standard frozen coefficient theory, we may assume that the equation is linear with constant coefficients and the flow is subsonic, which is the case in farfield. Thus we consider

$$
\begin{gathered}
2 \phi_{x t}=K^{*} \phi_{x x}+\phi_{y y}, \quad K^{*}>0 \\
|x| \leq a,|y| \leq b
\end{gathered}
$$

Let $(\tau, \xi, \eta)$ be the dual of $(t, x, y)$, then the dispersion relation of (4.1) becomes

$$
\sigma(\tau, \xi, \eta)=2 \tau \xi-K^{*} \xi^{2}-\eta^{2}=0
$$

We first consider the sidewalls.

\section{Sidewall condition}

The $y$-component of the group velocity of the plane wave $e^{i(\tau t+\xi x+\eta y)}$ is equal to

$$
V_{y}=\frac{\sigma_{\eta}}{\sigma_{\tau}}=-\frac{2 \eta}{2 \xi}=-\frac{\eta}{\xi}
$$


Dividing through (4.2) by $\xi^{2}$, we have

$$
\left(\frac{\eta}{\xi}\right)^{2}=2 \frac{\tau}{\xi}-K^{*}
$$

From this relation, we are able to determine the range of group velocity. Since the righthand side can be made arbitrarily large, the group velocity is unbounded, i.e.,

$$
V_{y} \in(-\infty,+\infty)
$$

Even though the propagating speed in the $y$-direction may be infinity, it can be justified to consider only the group velocities in a bounded interval, for the following reasons.

The propagating speed of a plane wave in the $y$-direction becomes infinity only if the wave is parallel to $x$-axis, i.e., $\xi=0$. In this case, as is easy to verify, the propagating speed downstream is also infinite and is much faster than the $y$-component because

$$
\frac{V_{y}}{V_{x}} \rightarrow 0 \text { as } \xi \rightarrow 0
$$

This implies that the disturbance with infinite $y$-direction speed will actually strike the downstream boundary first. So in designing a sidewall boundary condition, we need only to consider those waves with finite propagating speed in the $y$-direction. Thus, we may assume there is a $\beta>0$ such that

$$
V_{y} \in[-\beta, \beta]
$$

For a given $(\tau, \xi) \in \mathbb{R}^{2}$, equation (4.2) has two roots $\eta^{+}=-\eta^{-}$, corresponding to the two travelling wave modes, with

$$
0<V_{y}\left(\eta^{+}\right)=-V_{y}\left(\eta^{-}\right) .
$$

Thus as a direct consequence of Theorem 3.3 , an $\mathcal{L}_{\varepsilon}^{\infty}$-best boundary condition of order $d$ at sidewall $y=b$ (resp., $y=-b$ ) must have the form

$$
Q_{d}(x)=\prod_{j=1}^{d}\left(x-v_{j}\right),
$$


with $v_{j} \in[\varepsilon, \beta]$ (resp., $v_{j} \in[-\beta,-\varepsilon]$ ), $\varepsilon>0$. If the reflection coefficient of (4.4) is denoted by $R_{Q}$, then it follows from Theorem 3.5 that

$$
R_{Q}(\tau, \xi)<1 \quad \text { for all }(\tau, \xi) \in \mathcal{S}_{\varepsilon}
$$

where $\mathcal{S}_{\varepsilon}$ is defined in the Definition 3.1.

The theory of section 3 does not assure that the boundary condition given by (4.4) is well-posed; it has to be determined by the criterion given at the beginning of section 3 . In order to prove the well-posedness, we need only to show that each factor in (4.4) yields a well-posed boundary condition. Thus, let us consider the factor $\left(x-v_{j}\right)$. The dispersion relation of the corresponding boundary condition is

$$
-\frac{\eta}{\xi}-v_{j}=0
$$

or

$$
\eta+v_{j} \xi=0
$$

Let us consider the mutual solutions of (4.2) and (4.5). For any $\xi \in \mathbb{R}$, the solution $\eta$ of (4.5) is always real, thus an eigenvalue does not exist. Also, for any $\xi \in \mathbb{R},\left(\xi, \eta^{+}\right)$ will never satisfy $(4.5)$ since $v_{j}<0$. This implies there is also no generalized eigenvalues, so the boundary condition is well-posed.

From this, the best boundary condition at the sidewalls should have the form

$$
\prod_{j=1}^{d}\left(\frac{\partial}{\partial y}+v_{j} \frac{\partial}{\partial x}\right) \phi=0
$$

with $v_{j}<0$ for $y=-b$ and $v_{j}>0$ for $y=b$. The parameters $v_{j}$ 's must be tuned to make the reflection coefficient small. Details in the determination of parameters will be given in section 5. In general, if we know a priori the main interior disturbances propagate with certain speeds in the $y$-direction, these disturbances can be exactly annihilated by tuning $v_{j}$ to those speeds. 
In [8], $v_{1}=-\sqrt{K^{*}}$ was chosen for the first-order condition, which annihilates exactly the waves travelling in the $y$-direction only, i.e., with zero speed in the $x$-direction.

\section{Upstream condition}

The $x$-direction of group velocity is given by

$$
V_{x}=\frac{\sigma_{\xi}}{\sigma_{\tau}}=-K^{*}+\frac{\tau}{\xi}
$$

By employing the previous techniques, we find that

$$
V_{x} \in\left[-\frac{K^{*}}{2},+\infty\right)
$$

It follows from Theorem 3.3 that the $\mathcal{L}_{e}^{\infty}$-best boundary condition of order $d$ at $x=-a$ has the form (which is well-posed):

$$
\prod_{j=1}^{d}\left(\frac{\partial}{\partial t}-\left(K^{*}+v_{j}\right) \frac{\partial}{\partial x}\right) \phi=0
$$

where $v_{j} \in\left[-\frac{K^{*}}{2},-\varepsilon\right]$. Its reflection coefficient is smaller than 1 by Theorem 3.5. For the first-order condition, $v_{1}=-\frac{K^{*}}{2}$ was chosen in [8], the corresponding boundary condition annihilating waves travelling at the fastest speed upstream.

The second-order condition of $[8]$ is given by

$$
\phi_{x t}-\frac{2}{K^{*}} \phi_{t t}+\frac{1}{2} \phi_{y y}=0
$$

which is also a special case of (4.7). In fact, if one solves for $\phi_{y y}$ from (4.1) and substitutes it into the above equation, the result is

$$
2 \phi_{x t}-\frac{2}{K^{*}} \phi_{t t}-\frac{K^{*}}{2} \phi_{x x}=0
$$

This reduces to (4.7) with $v_{1}=v_{2}=-\frac{K^{*}}{2}$. 


\section{Downstream condition}

At the downstream $x=a$, the $\mathcal{L}_{\varepsilon}^{\infty}$-best boundary condition also has the form (4.7) but with $v_{j}>0$. Condition (4.7) is well-posed at $x=a$ if $v_{j}>0$, and its reflection coefficient is equal to

$$
R(\tau, \eta)=\prod_{j=1}^{d}\left(\frac{v_{j}-\left(K^{*}+v_{j}\right) \sqrt{1-K^{*}\left(\frac{\eta}{\tau}\right)^{2}}}{v_{j}+\left(K^{*}+v_{j}\right) \sqrt{1-K^{*}\left(\frac{\eta}{\tau}\right)^{2}}}\right) .
$$

It is clear that $R(\tau, \eta)<1$ if $\sqrt{1-K^{*}\left(\frac{\eta}{\tau}\right)^{2}} \neq 0$, i.e., $V_{x}(\xi) \neq 0$.

Since the interior disturbance might contain certain modes which have infinite downstream speed, one of the parameters can be tuned to annihilate these wave modes, e.g., $v_{1}=+\infty$ in the first-order condition. In this case, the reflection coefficient becomes

$$
R_{1}(\tau, \eta)=\frac{1-\sqrt{1-K^{*}\left(\frac{\eta}{\tau}\right)^{2}}}{1+\sqrt{1-K^{*}\left(\frac{\eta}{\tau}\right)^{2}}}
$$

and the corresponding boundary condition is obtained by taking the limit $v_{1} \rightarrow+\infty$ :

$$
\frac{\partial}{\partial x} \phi=0, \quad \text { at } x=a .
$$

This is exactly the boundary condition given in [8]. For higher order, we propose

$$
\prod_{j=1}^{d-1}\left(\frac{1}{K^{*}+v_{j}} \frac{\partial}{\partial t}-\frac{\partial}{\partial x}\right) \frac{\partial}{\partial x} \phi=0
$$

\subsection{Equation of Full Frequencies}

We now consider the full frequency transonic small disturbance equation

$$
\phi_{t t}+2 \phi_{x t}=K^{*} \phi_{x x}+\phi_{y y}
$$

Its dispersion relation is

$$
\sigma(\tau, \xi, \eta)=\tau^{2}+2 \xi \tau-K^{*} \xi^{2}-\eta^{2}=0
$$

\section{Sidewall condition}


The $y$-component of group velocity is equal to

$$
V_{y}=\frac{\sigma_{\eta}}{\sigma_{\tau}}=-\frac{\eta}{\tau+\xi}
$$

It can be shown that

$$
V_{y} \in[-1,1]
$$

and the two travelling wave modes satisfy

$$
\eta^{+}=-\eta^{-} \quad \text { and } \quad\left|V_{y}\left(\eta^{+}\right)\right|=\left|V_{y}\left(\eta^{-}\right)\right|,\left|\sigma_{\tau}\left(\eta^{+}\right)\right|=\left|\sigma_{\tau}\left(\eta^{-}\right)\right|
$$

Applying Theorem 3.3 and Theorem 3.5 , the $\mathcal{L}_{\varepsilon}^{\infty}$-best condition has the form

$$
Q^{*}(x)=\prod_{j=1}^{d}\left(x-v_{j}\right),
$$

$v_{j} \in[-1,-\varepsilon]$ for $y=-b$ and $v_{j} \in[\varepsilon, 1]$ for $y=b$. Furthermore $R_{Q^{*}}<1$. Therefore, the sidewall conditions may be chosen as

$$
\prod_{j=1}^{d}\left(\frac{\partial}{\partial y}+v_{j} \frac{\partial}{\partial t}+v_{j} \frac{\partial}{\partial x}\right) \phi=0 .
$$

The condition is well-posed.

\section{Upstream and downstream conditions}

From the dispersion relation (4.9), we find

$$
V_{x}=1-\left(K^{*}+1\right) \frac{\xi}{\tau+\xi}
$$

and

$$
V_{x} \in\left[1-\sqrt{1+K^{*}}, 1+\sqrt{1+K^{*}}\right]
$$

It follows from Theorem 3.3 that the absorbing boundary conditions can be taken as

$$
\prod_{j=1}^{d}\left(\left(1-v_{j}\right) \frac{\partial}{\partial t}-\left(K^{*}+v_{j}\right) \frac{\partial}{\partial x}\right) \phi=0
$$


where $v_{j} \in\left[1-\sqrt{1+K^{*}},-\varepsilon\right]$ for upstream condition and $v_{j} \in\left[\varepsilon, 1+\sqrt{1+K^{*}}\right]$ for downstream condition.

It can be verified directly that the reflection coefficients associated with (4.10) for both upstream and downstream are strictly less than 1 , except for the mode with zero $x$-component group velocity.

\section{NUMERICAL COMPUTATIONS}

In this section, we discuss the numerical implementation and present some computational results involving the absorbing boundary conditions derived in the last section. For the purpose of illustrating the efficiency of these conditions, we compare the computational results obtained by using the boundary conditions of this paper with those developed by Engquist and Majda in [8], both applied to the linear equation (4.1) in the region $\Omega=[-1,1] \times[-1,1]$. We note that the first-order conditions derived from the last section are the same as those derived in [8].

Specifically, we compare the following sets of conditions:

\section{Upstream:}

First-order:

$$
\phi_{t}+v \phi_{x}=0
$$

Second-order:

$$
\begin{aligned}
\phi_{x t}-\frac{2}{K^{*}} \phi_{t t}+\frac{1}{2} \phi_{y y} & =0 \\
\left(\frac{\partial}{\partial t}-\left(K^{*}+v_{1}\right) \frac{\partial}{\partial x}\right)\left(\frac{\partial}{\partial t}-\left(K^{*}+v_{2}\right) \frac{\partial}{\partial x}\right) \phi & =0
\end{aligned}
$$

\section{Downstream:}

First-order:

$$
\phi_{x}=0
$$


Second-order:

$$
\begin{aligned}
\phi_{x x} & =0 \\
\left(\frac{1}{K^{*}+v} \frac{\partial}{\partial t}-\frac{\partial}{\partial x}\right) \frac{\partial}{\partial x} \phi & =0
\end{aligned}
$$

Sidewall $(y=1)$ :

First-order:

$$
\phi_{y}+v \phi_{x}=0
$$

Second-order:

$$
\begin{aligned}
\phi_{t y}+r_{2} \phi_{x y}+r_{1} \phi_{x t} & =0 \\
\left(\frac{\partial}{\partial y}+v_{1} \frac{\partial}{\partial x}\right)\left(\frac{\partial}{\partial y}+v_{2} \frac{\partial}{\partial x}\right) \phi & =0
\end{aligned}
$$

\subsection{Parameters}

Theoretically, the parameters in the above boundary conditions should be determined by the minimization properties stated in the definitions 3.1 and 3.2. Such process, however, is hardly necessary in practice, nor will it always yield the best result for all problems, because the definition of the $\mathcal{L}_{\varepsilon}^{\infty}$ - or $\mathcal{L}^{2}$-best conditions itself depends on the arbitrary choice of $\varepsilon$ or the weight $\rho$. In the present study, we use the following procedure in which the parameters can be easily determined.

For the upstream conditions, the parameters must satisfy $v \in\left[-\frac{K^{*}}{2}, 0\right)$. The firstorder condition is tuned to the waves travelling most rapidly upstream by letting $v=-\frac{K^{*}}{2}$. In the second-order condition (5.1), the two parameters are chosen as $v_{1}=-\frac{K^{*}}{2}$ and $v_{2}=-\frac{K^{*}}{4}$, the mid-point of $\left[-\frac{K^{*}}{2}, 0\right)$.

The parameters in the downstream conditions must be in $(0,+\infty)$, which is unbounded. Howcver, a transformation exists to map $(0,+\infty)$ to the bounded interval $(0,1)$, 
hence the similar method as above can be used. Recall from section 4 that a factor of reflection coefficient of the downstream condition is

$$
\begin{aligned}
R_{j} & =\frac{v_{j}-\left(K^{*}+v_{j}\right) \sqrt{1-K^{*}\left(\frac{\eta}{\tau}\right)^{2}}}{v_{j}+\left(K^{*}+v_{j}\right) \sqrt{1-K^{*}\left(\frac{\eta}{\tau}\right)^{2}}}, \\
& =\frac{\frac{v_{j}}{\left(K^{*}+v_{j}\right)}-\sqrt{1-K^{*}\left(\frac{\eta}{\tau}\right)^{2}}}{\frac{v_{j}}{\left(K^{*}+v_{j}\right)}+\sqrt{1-K^{*}\left(\frac{\eta}{\tau}\right)^{2}}}
\end{aligned}
$$

where $\frac{v_{j}}{K^{*}+v_{j}} \in(0,1)$.

Thus the first-order downstream condition is obtained by letting $\frac{v}{K^{*}+v}=1$, i.e., $v=+\infty$. The remaining parameter $v$ in the second-order condition (5.2) is determined by requiring $\frac{v}{K^{*}+v}=\frac{1}{2}$, therefore, $v=K^{*}$.

In the case of sidewall, we have to deal with the genuine unbounded interval. For the reasons mentioned in section $4, v=\sqrt{K^{*}}$ in the first-order sidewall $(y=1)$ condition. Parameters $v_{1}, v_{2}$ in (5.3) are determined by minimizing the integral

$$
\int_{0}^{\beta} e^{-\alpha\left(x-\sqrt{K^{*}}\right)^{2}} R_{2}(x)^{2} d x
$$

where, by section $4, R_{2}=\left(\frac{x-v_{1}}{x+v_{1}}\right)\left(\frac{x-v_{2}}{x+v_{2}}\right)$.

By a change of variables, the above integral becomes

$$
\sqrt{K^{*}} \int_{0}^{\tilde{\beta}} e^{-\tilde{\alpha}(x-1)^{2}}\left(\frac{x-\tilde{v}_{1}}{x+\tilde{v}_{1}}\right)^{2}\left(\frac{x-\tilde{v}_{2}}{x+\tilde{v}_{2}}\right)^{2} d x
$$

where $\tilde{\beta}=\frac{\beta}{\sqrt{K^{*}}}, \tilde{\alpha}=\alpha K^{*}$ and $\tilde{v}_{j}=\frac{v_{j}}{\sqrt{K^{*}}} . \tilde{v}_{1}$ and $\tilde{v}_{2}$ can be computed by a numerical integration and optimization algorithm. For $\tilde{\beta}=10$. and $\tilde{\alpha}=0.1$, we found, correct to the first digit, $\tilde{v}_{1}=3.0$ and $\tilde{v}_{2}=0.4$, hence $v_{1}=3.0 \sqrt{K^{*}}$ and $v_{2}=0.4 \sqrt{K^{*}}$ in (5.3).This particular choice of parameters yields a reflection coefficient $R_{2} \leq 0.05$ for all wave modes with the group velocities in the range $[0.25,5.0]$ (see [15]).

Finally, in this study, $r_{1}=6.4641 \sqrt{K^{*}}$ and $r_{2}=5.4641 K^{*}$ in (E \& M) second-order condition, according to one of the guidelines of [8]. 


\subsection{Discretization}

Equation (4.1) is discretized by a semi-implicit scheme described in [2], where the $x$-direction is treated explicitly and the $y$-direction is kept implicit. The resulting difference equation are solved by marching both in the $x$ - and $t$-direction. At a given time level, tridiagonal equations are solved along the $y$-directions, successively marching in the downstream direction. For stability, the C.F.L. condition $K^{*} \frac{\Delta t}{\Delta x} \leq 2$ must be satisfied. The scheme is nondissipative when $K^{*} \frac{\Delta t}{\Delta x}=2$.

For the first-order boundary conditions and the second-order Engquist-Majda (E \& M) boundary conditions, we use exactly the discretization suggested in [8]. Since each factor in (5.1), and (5.3) is in the form of the first-order operator of the corresponding first-order boundary condition, the discretizations of (5.1) and (5.3) are obtained by applying twice the difference operators which discretize the corresponding first-order boundary conditions of Engquist and Majda. For example, in [8], the boundary condition

$$
\phi_{y}+v \phi_{x}=0
$$

is discretized by

$$
\left\{\frac{I-K^{-1}}{\Delta y}\left(I+J^{-1}\right)+v \frac{I-J^{-1}}{\Delta x}\left(I+K^{-1}\right)\right\} \phi_{j, k+1}^{n+1}=0,
$$

where $J, K$ are shift operators in $x$ and $y$ respectively, $J \phi_{j, k}^{n}=\phi_{j+1, k}^{n}, K \phi_{j, k}^{n}=\phi_{j, k+1}^{n}$. By applying this twice, the difference equation for (5.3) becomes

$$
\begin{aligned}
\left\{\frac{I-K^{-1}}{\Delta y}\left(I+J^{-1}\right)\right. & \left.+v_{1} \frac{I-J^{-1}}{\Delta x}\left(I+K^{-1}\right)\right\} \\
& \times\left\{\frac{I-K^{-1}}{\Delta y}\left(I+J^{-1}\right)+v_{2} \frac{I-J^{-1}}{\Delta x}\left(I+K^{-1}\right)\right\} \phi_{j, k+1}^{n+1}=0 .
\end{aligned}
$$

Condition (5.2) is discretized by an explicit scheme, backward in $x$.

The stability of these difference schemes can be determined by the GKS theory [11], or by numerical experiments. Because of the factorization form, the difference schemes 
for the higer-order boundary conditions are stable if and only if, by the GKS theory, the schemes for the corresponding first-order conditions are stable. The discretization of the first-order boundary conditions is discussed in detail in [8], and although no analysis has been attempted, these schemes are found to be stable by numerical experiments, even for the nonlinear problem.

Since the interior equation is solved line by line marching in the downstream direction, the boundary condition (5.4) which requires values of $\phi$ at $j-1, j-2$ cannot be applied to the immediate neighboring point of the corner of the upstream and the sidewall boundaries. This difficulty is overcome by using the following condition at the upstream corner:

$$
\alpha(x)\left(\frac{\partial}{\partial y}+v \frac{\partial}{\partial x}\right) \phi+(1-\alpha(x))\left(\frac{\partial}{\partial y}+v_{1} \frac{\partial}{\partial x}\right)\left(\frac{\partial}{\partial y}+v_{2} \frac{\partial}{\partial x}\right) \phi=0,
$$

where $0 \leq \alpha \leq 1$. $\alpha$ decreases smoothly from $\alpha=1$ at the upstream boundary to 0 . In our study, the transition region of $\alpha$ from 1 to 0 extends to 2 grid points.

There is no difficulty at the downstream corners, since these corner points are not involved in the computation.

\subsection{Energy}

In the following computations, we will use the energy of solutions as a means of measurement. The energy

$$
\begin{array}{r}
E(n)=\sum_{j, k} K^{*}\left\{\frac{\phi_{j+1, k+1}^{n}+\phi_{j+1, k}^{n}-\phi_{j, k+1}^{n}-\phi_{j, k}^{n}}{2 \Delta x}\right\}^{2} \Delta x \Delta y \\
+\sum_{j, k}\left\{\frac{\phi_{j+1, k+1}^{n}+\phi_{j, k+1}^{n}-\phi_{j+1, k}^{n}-\phi_{j, k}^{n}}{2 \Delta y}\right\}^{2} \Delta x \Delta y
\end{array}
$$

is the discrete version of

$$
E(t)=\iint_{|x| \leq 1}\left(K^{*} \phi_{x}^{2}+\phi_{y}^{2}\right) d x d y
$$

It is casy to verify that if $\phi$ satisfies (4.1) in $|x|<1$ and $|y|<1$, and the perfectly reflecting boundary conditions

$$
\phi=0, \quad \text { at } \quad x= \pm 1 \text { and } y= \pm 1
$$


then the energy of solutions is conserved, that is,

$$
\frac{d}{d t} E(t) \equiv 0, \quad \text { for all } t
$$

In order to examine the effects of each absorbing boundary condition, without being influenced by the presence of the other boundary conditions, we will use a strategy where the absorbing boundary condition will be prescribed at one boundary only in each experiment, and the perfectly reflecting boundary condition (5.6) will be imposed on the remaining boundaries. In the first group of calculations, the energy (5.5) of solutions in the domain $\Omega=[-1,1] \times[-1,1]$ will be computed. Therefore, the rate of decrease of the energy will demonstrate the ability of the test boundary condition to radiate the energy away from the computational domain, because by (5.7), energy of the solution will be totally reflected back from the other three perfectly reflecting boundaries.

In all computations, $K^{*}=1$ is used in the equation (4.1), and the region $\Omega=$ $[-1,1] \times[-1,1]$ is covered by a $60 \times 40$ uniform mesh, with a $C F L$ condition of 1.997. For an initial value, we use a pulse of compact support, a piece of radially symmetrical sine function. Energy of solutions is calculated after every 10 time steps, with a total computation of 230 time steps. The initial pulse will soon spread out in a parabolic wavefront [8], and total reflections will occur at the three perfectly reflecting boundaries, so after some time, the disturbance that strikes the test boundary will consist of wave packets with a fairly large spectrum of frequencies and wave numbers.

Figure 1 shows the energy $E^{0}(n)$, given by (5.5), of the solution of (4.1) with the perfectly reflecting boundary conditions (5.6) at different time steps. The decrease in the encrgy is due to the dissipation of the difference scheme. In order to eliminate the effect of this dissipation in our study, the energy of the solutions for other calculations will all be scaled by $E^{0}(n)$, i.e., the energy

$$
c(n)=\left(\frac{E(n)}{E^{0}(n)}\right) \times 100,
$$


will be used for other calculations. Therefore $e(n) \equiv 100$ if the perfectly reflecting boundary condition $\phi=0$ is prescribed on all boundaries.

The results of calculations involving boundary conditions on the upstream, downstream and sidewall $(y=1)$ boundaries are presented by graphs in Fig. 2, Fig. 3 and Fig. 4, respectively. The curves of the energy $e(n)$ all follow one pattern: during the short period of the initial time (about 30 time steps), the three (one first-order and two second-order) boundary conditions produce roughly the same results, but they differ significantly after a long time period (after about 130 time steps).

In the second group of calculations, we measure the energy of the reflected waves of different absorbing boundary conditions. The reflected wave is obtained by comparing the solution of an absorbing boundary condition with the free-space solution which is calculated in a larger computational area. For example, in the computations involving upstream boundary conditions, the free-space solution is calculated in the region $\Omega \cup \bar{\Omega}$ as illustrated in Fig. 5. Then an absorbing boundary condition is used on the boundary $x=-1$, and the corresponding solution is computed in the region $\Omega$. The difference of these two solutions in $\Omega$ can be considered as the reflection caused by the absorbing boundary condition. Similar methods are used for the downstream and the sidewall boundaries.

The results for the energy of reflected waves are shown in Fig. 6, Fig. 7 and Fig. 8 . As before, the energy is calculated at every 10 time steps. In each graph, the curves are scaled so that the maximum energy in the first-order boundary condition is 100 .

These numerical results clearly show the improved performance of the second-order absorbing boundary conditions over the first-order conditions. In long time computations, the second-order conditions (5.1), (5.2) and (5.3) produce less reflections than the corresponding $(E \& M)$ conditions. However, the second-order $(E \& M)$ conditions generate smaller reflections during the very short period of the initial time because they are tuned to the waves travelling most rapidly. These observations agree with the analysis of the reflection coefficients given in the section 4 . 


\section{Acknowledgment}

We thank Dr. B.H.K. Lee of the National Aeronautical Establishment (Ottawa, Canada) for his interest in and support of this research work. 


\section{REFERENCES}

[1] W.F. Ballhaus and P.M. Goorjan, AIAA Paper, 77 (1977).

[2] W.F. Ballhaus and J.O. Bridgeman, "Special course on unsteady aerodynamics", AGARD Report No. 679, (1980).

[3] A. Bamberger, B. Engquist, L. Halpern and P. Joly, SIAM J. Appl. Math., 48, 99 (1988).

(4] A. Bamberger, B. Engquist, L. Halpern and P. Joly, SIAM J. Appl. Math., 48, 129 (1988).

[5] A. Bayliss and E. Turkel, Commun. Pure Appl. Math., 33 , 707 (1980).

[6] B. Engquist and A. Majda, Math. Comput., 31, 629 (1977).

[7] B. Engquist and A. Majda, Commun. Pure Appl. Math., 32, 313 (1979).

[8] B. Engquist and A. Majda, J. Comput. Phys., 40, 91 (1981).

[9] C. Grosch and S. Orszag, J. Comput. Phys., 25, 273 (1977).

[10] B. Gustafsson and H.O. Kreiss, J. Comput. Phys., 30, 333 (1979).

[11] B. Gustafsson, H.-O. Kreiss and A. Sundstrom, Math. Comput., 26, 649 (1972).

[12] G.W. Hedstrom, J. Comput. Phys., 30, 222 (1979).

13] R.L. Higdon, Math. Comput., 47, 437 (1986).

[14] R.L. Higdon, SIAM Rev., 28, 177 (1986).

[15] H. Jiang, Ph.D. Dissertation, (1988, in preparation).

[16] K.R. Kelly, R.M. Alford, S. Treifel and R.W. Ward, Proc. Roy. Irish Acad. Conf. on Numerical Analysis, (Academic Press, London \& New York, 1974), p. 7.

[17] H.O. Kreiss, Commun. Pure Appl. Math., 23, 277 (1970).

[18] D.J. Newman, Michigan Math., 11, 11 (1964).

(19) L. Nirenberg, Lectures on linear partial differential equations, "C.B.M.S Regional Conf. Ser. in Math., no. 17", (Ameri. Math. Soc., Providence, R.I. , 1973).

[20] P.J. Roache, Computational fluid dynamics, (Hermosa Press, Albuquerque, N.M., 1972).

[21] R. Sakamoto, Hyberbolic boundary value problems, (Cambridge university press, 1978).

[22] K.W. Thompson, J. Comput. Phys., 68, 1 (1987).

[23] L.N. Trefethen, Ph.D. Dissertation,(1982).

[24] L.N. Trefethen and L. Halpern, Math. Comput., 47, 421 (1986).

[25] L. Wagatha, Numer. Math., 42, 51 (1983).

[26] G.B. Whitham, Linear and Nonlinear Waves, (Wiley-Interscience, New York, 1974), P. 383. 

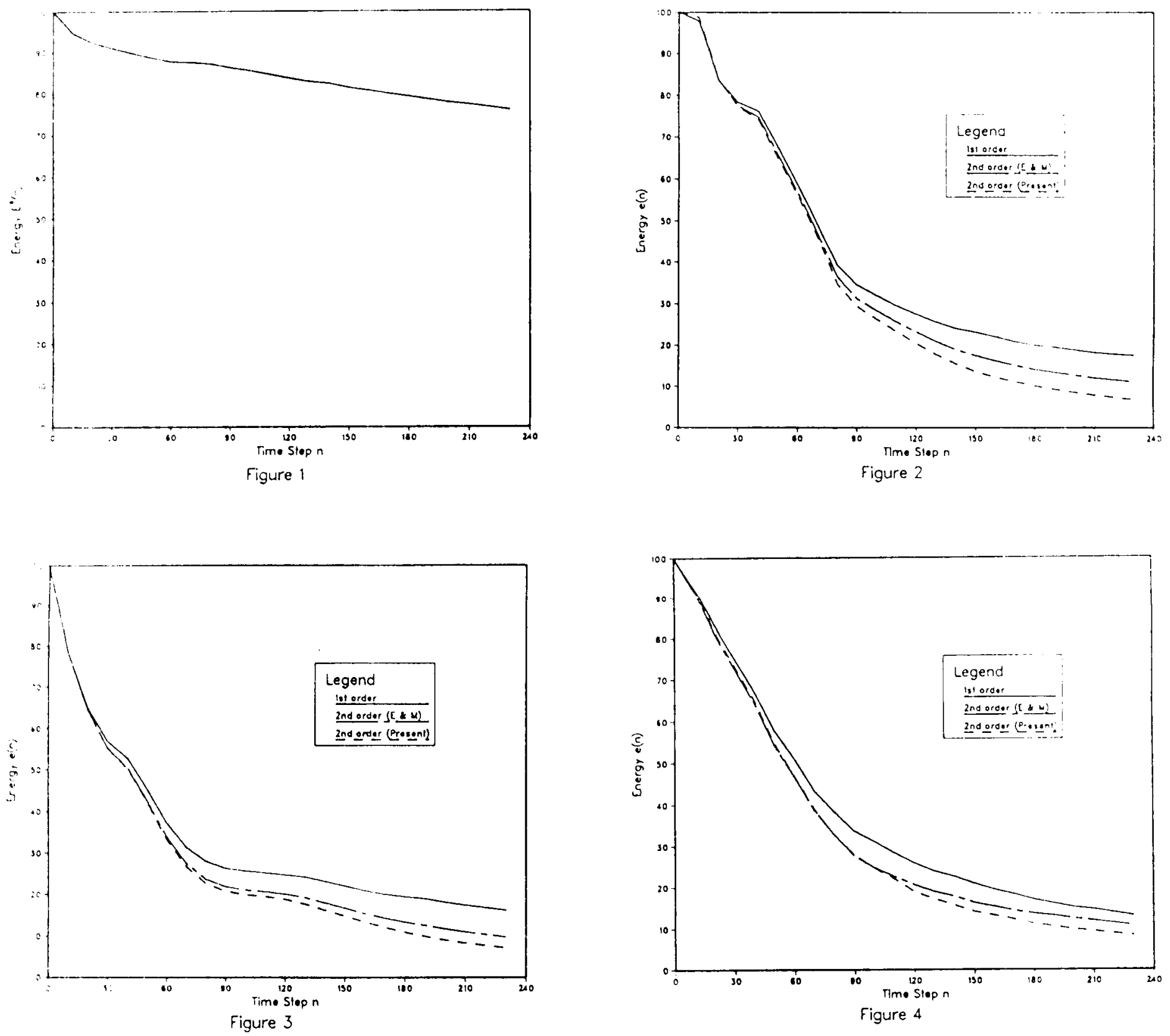

Fig. 1: Energy of the solution, with the perfectly reflecting condition on all four sides. The decrease in the energy is due to the dissipation of the scheme.

Fig. 2: Energy of the solutions: Upstream boundary. $v_{1}=-\frac{K^{*}}{2}, v_{2}=-\frac{K^{*}}{4}$ in condition (5.1).

Fig. 3: Energy of the solutions: Downstream boundary. $v=K^{*}$ in condition (5.2).

Fig. 4: Energy of the solutions: Sidewall $(y=1)$ boundary. $r_{1}=6.4641 \sqrt{K^{*}}, r_{2}$ $5.4641 K^{*}$ in $(\mathrm{E} \& \mathrm{M})$, and $v_{1}=3.0 \sqrt{K^{*}}, v_{2}=0.4 \sqrt{K^{*}}$ in condition (5.3). 


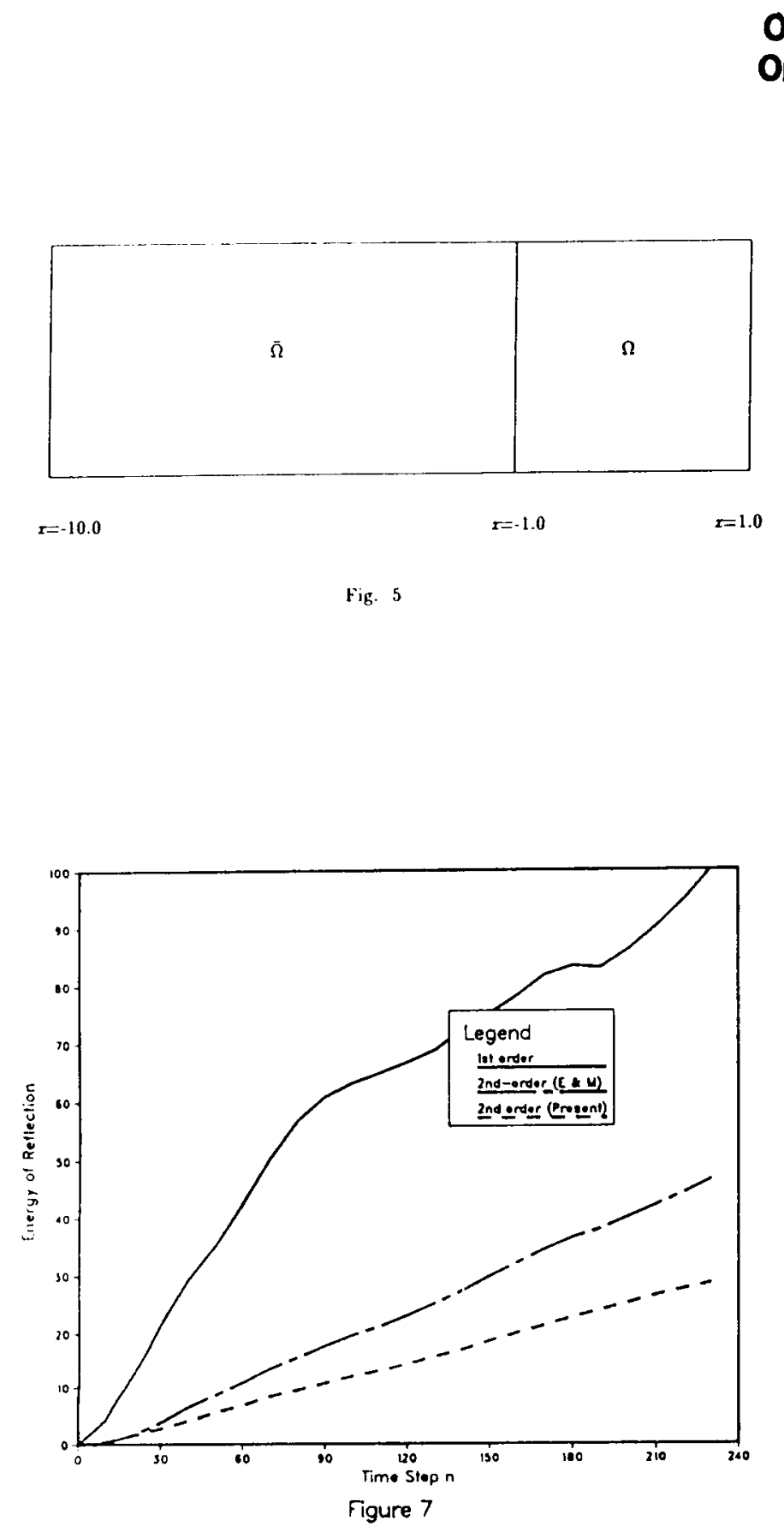

ORIGINAL PAGE IS

OF POOR QUALITY
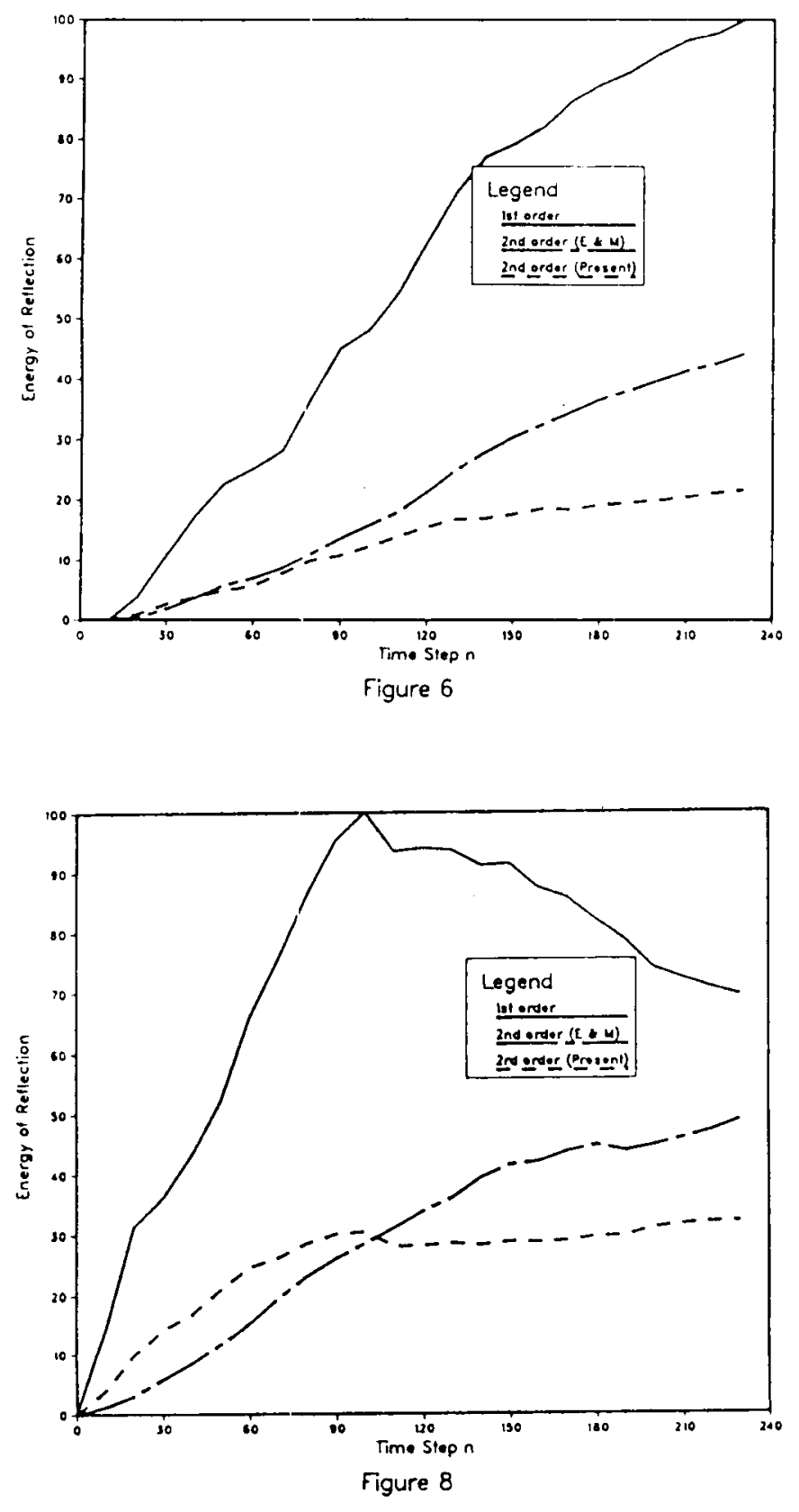

Fig. 5: Large computational area for free-space solution calculation: Upstream boundary.

Fig. 6: Energy of the reflected waves: Upstream boundary.

Fig. 7: Energy of the reflected waves: Downstream boundary.

Fig. 8: Energy of the reflected waves: Sidewall $(y=1)$ boundary. 


\begin{tabular}{|c|c|c|c|c|}
\hline & \multicolumn{4}{|c|}{ Report Documentation Page } \\
\hline 1. & Report No. & $\begin{array}{l}\text { NASA TM-102009 } \\
\text { ICOMP-89-7 }\end{array}$ & 2. Government Accession No. & 3. Recipient's Catalog No. \\
\hline \multirow[t]{3}{*}{4} & \multicolumn{3}{|c|}{ Title and Subtitle } & 5. Report Date \\
\hline & \multirow{2}{*}{\multicolumn{3}{|c|}{ Absorbing Boundary Conditions for Second-Order Hyperbolic Equations }} & April 1989 \\
\hline & & & & 6. Performing Organization Code \\
\hline \multirow[t]{4}{*}{$\overline{7}$} & \multicolumn{3}{|l|}{ Author(s) } & 8. Performing Organization Report No. \\
\hline & \multirow{3}{*}{\multicolumn{3}{|c|}{ Hong Jiang and Yau Shu Wong }} & E-4719 \\
\hline & & & & 10. Work Unit No. \\
\hline & & & & $505-62-21$ \\
\hline \multicolumn{4}{|c|}{ 9. Performing Organization Name and Address } & 11. Contract or Grant No. \\
\hline \multirow{2}{*}{\multicolumn{4}{|c|}{$\begin{array}{l}\text { National Aeronautics and Space Administration } \\
\text { Lewis Research Center } \\
\text { Cleveland. Ohio } 44135-3191\end{array}$}} & \\
\hline & & & & 13. Type of Report and Period Covered \\
\hline \multirow[t]{2}{*}{12.} & \multicolumn{3}{|c|}{ Sponsoring Agency Name and Address } & Technical Memorandum \\
\hline & \multicolumn{3}{|c|}{$\begin{array}{l}\text { National Aeronautics and Space Administration } \\
\text { Washington, D.C. } 20546-0001\end{array}$} & \begin{tabular}{|l} 
14. Sponsoring Agency Code \\
\end{tabular} \\
\hline \multicolumn{5}{|c|}{ 15. Supplementary Notes } \\
\hline & \multicolumn{4}{|c|}{$\begin{array}{l}\text { Hong Jiang, Department of Mathematics, University of Alberta, Edmonton, Canada; Yau Shu Wong, Institute for } \\
\text { Computational Mechanics in Propulsion, Lewis Research Center (work funded under Space Act Agreement } \\
\text { C99066G) and Department of Mathematics, University of Alberta, Edmonton, Canada. }\end{array}$} \\
\hline
\end{tabular}

A uniform approach to construct absorbing artificial boundary conditions for second-order linear hyperbolic equations is proposed. The nonlocal boundary condition is given by a pseudodifferential operator that annihilates travelling waves. It is obtained through the dispersion relation of the differential equation by requiring that the initial-boundary value problem admits the wave solutions travelling in one direction only. Local approximation of this global boundary condition yields an $n$ th-order differential operator. It is shown that the best approximations must be in the canonical forms which can be factorized into first-order operators. These boundary conditions are perfectly absorbing for wave packets propagating at certain group velocities. A hierarchy of absorbing boundary conditions is derived for transonic small perturbation equations of unsteady flows. These examples illustrate that the absorbing boundary conditions are easy to derive, and the effectiveness is demonstrated by the numerical experiments.

17. Key Words (Suggested by Author(s))

Absorbing boundary conditions Second-order hyperbolic equations Unsteady transonic small disturbance equations
18. Distribution Statement

Unclassified - Unlimited

Subject Category 64 